\title{
Analysis of nanostructure and nanochemistry by ASAXS: Accessing phase composition of oxyfluoride glass ceramics doped with $\mathrm{Er}^{3+} / \mathrm{Yb}^{3+}$
}

\author{
Sylvio Haas* and Armin Hoell \\ Institute of Applied Materials, Helmholtz-Zentrum Berlin für Materialien und Energie, \\ Albert-Einstein-Straße 15, D-12489 Berlin, Germany
}

Roman Wurth and Christian Rüssel

Otto-Schott-Institut für Glaschemie, Friedrich-Schiller-Universität Jena, Fraunhoferstraße 6, D-07743 Jena, Germany

Peter Boesecke

European Synchrotron Radiation Facility (ESRF), 6 Rue Jules Horowitz, BP 220, 38043 Grenoble Cedex 9, France

Ulla Vainio

HASYLAB at DESY, Notkestraße 85, D-22607 Hamburg, Germany

(Received 1 October 2009; revised manuscript received 25 January 2010; published 18 May 2010)

\begin{abstract}
Here, we describe the analysis of the nanostructure and average chemical compositions of each phase present in an oxyfluoride glass ceramic, which is composed of fluoride nanocrystals and an oxide glass matrix. The overall composition of the oxyfluoride glass ceramic as prepared is $21.1 \% \mathrm{SiO}_{2} \quad 6.5 \% \mathrm{~B}_{2} \mathrm{O}_{3} \quad 7.0 \% \mathrm{Al}_{2} \mathrm{O}_{3} \quad 21.0 \% \mathrm{PbF}_{2} \quad \begin{array}{lllll}14.3 \% \mathrm{CdF}_{2} & 11.0 \% \mathrm{YbF}_{3} & 0.5 \% \mathrm{ErF}_{3} & 11.0 \% \mathrm{PbO} & 7.6 \% \mathrm{CdO}\end{array}$ (mole \%). Nanocrystals begin to grow at temperatures above the glass transformation temperature at $678 \mathrm{~K}$ as observed by $\mathrm{x}$-ray diffraction. We report results from anomalous small-angle $\mathrm{x}$-ray scattering taken at energies of $\mathrm{x}$-ray absorption edges of $\mathrm{Er}, \mathrm{Yb}, \mathrm{Pb}$, and $\mathrm{Cd}$. By nonlinear regression of the scattering curves obtained from different edges simultaneously, the nanocrystals were found to be describable as polydisperse spheroids. The length of the smaller axis was found to be $6.4 \pm 1.4 \mathrm{~nm}$ while the larger axis was found to be $17.7 \pm 3.9 \mathrm{~nm}$. By analyzing the scattering contrast as a function of the x-ray energy we found cadmium only in the glass matrix.
\end{abstract}

DOI: 10.1103/PhysRevB.81.184207

PACS number(s): 61.05.cf, 81.05.Je, 81.07.-b

\section{INTRODUCTION}

Recently, a comprehensive review about fundamentals and prospects of upconversion materials has been published by Auzel. ${ }^{1}$ A large group of possible upconversion materials mentioned are oxyfluoride nanoglass ceramics (NGC), which have been studied extensively in the past. ${ }^{2-5}$ Depending on doping with lanthanide or transition-metal ions, the wavelength of the fluorescence emission of NGC can be adjusted and hence they are very attractive for a large number of applications. ${ }^{6,7}$ Oxyfluoride glasses doped with lanthanide ions such as $\mathrm{Er}^{3+}$ and $\mathrm{Yb}^{3+}$ have been rapidly emerging as promising materials for optoelectronic devices like fiber amplifiers. ${ }^{8-11}$ The ability of these glasses to transform nearinfrared radiation into visible and ultraviolet light, NIR to VIS/UV upconversion process, is of great interest due to possible applications in new solid-state lasers and luminescent bioassays. In the present work, we are focusing on the nanostructure determination of an NGC made of $\mathrm{SiO}_{2}, \mathrm{~B}_{2} \mathrm{O}_{3}$, $\mathrm{Al}_{2} \mathrm{O}_{3}, \mathrm{PbO}, \mathrm{CdO}, \mathrm{PbF}_{2}, \mathrm{CdF}_{2}$, and $M \mathrm{~F}_{3}(M=\mathrm{Er}, \mathrm{Yb})$. Wang and Ohwaki ${ }^{12}$ reported results from $\mathrm{x}$-ray diffraction (XRD) and fluorescence spectra analysis of a similar system. They suggested that $\mathrm{Yb}^{3+}$ and $\mathrm{Er}^{3+}$ ions are preferentially segregated from the precursor glass and dissolved into $\mathrm{Pb}_{x} \mathrm{Cd}_{1-x} \mathrm{~F}_{2}$ microcrystallites upon heat treatment. Kukkonen et al. ${ }^{13}$ reported a different crystalline phase based on the analysis of XRD, high-resolution transmission electron microscopy (HRTEM), and energy-dispersive x-ray spectroscopy (EDX).
The EDX analysis showed that $\mathrm{Er}^{3+}$ had preferentially segregated into the fluorite structure phase and that the phase is $\mathrm{PbF}_{2}$ rather than $\mathrm{Pb}_{x} \mathrm{Cd}_{1-x} \mathrm{~F}_{2}$ solid solution as previously supposed by Wang. The reported EDX spectra ${ }^{13}$ can give qualitatively the composition of the crystalline and glass matrix phases. The EDX spectrum for the crystalline phase shows some artificial peaks. Kukkonen associated the occurrence of these artifacts with contamination from the glue used while preparing the TEM samples.

Small-angle x-ray scattering (SAXS) is a method widely used in the analysis of internal nanoscale structures. ${ }^{14}$ The technique provides a number of structural parameters such as sizes, volume fraction, and shape of particles, as well as their correlations in the case of a denser particle system. In the case of a two phase system the differential scattering cross section $d \sigma(q) / d \Omega$ of a sample is proportional to the square of the electron-density difference between particle and dispersion medium (i.e., scattering contrast),

$$
\frac{d \sigma}{d \Omega}(q) \propto \Delta \rho^{2}=\left(\eta_{p}-\eta_{m}\right)\left(\eta_{p}-\eta_{m}\right)^{*}
$$

Here, $q$ means the scattering vector length, defined as $q$ $=4 \pi \sin (\theta) / \lambda . \theta$ is the half scattering angle and $\lambda$ the wavelength. The $\eta_{p}$ and $\eta_{m}$ in Eq. (1) are the electron densities of the corresponding phase (particle $=\mathrm{p}$ and dispersion medium $=\mathrm{m})$. In addition to SAXS allows the anomalous SAXS (ASAXS) element specific contrast variation between 


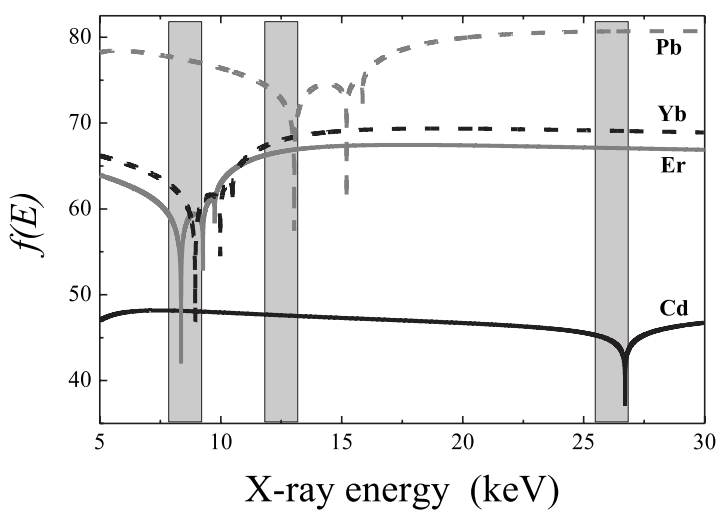

FIG. 1. The energy dependency of the atomic scattering factors (pure elements) of $\mathrm{Pb}, \mathrm{Er}, \mathrm{Yb}$, and $\mathrm{Cd}$ are shown. Close to X-ray absorption edges the values decrease up to $20 \%$ of the normal value (atomic number). Energies in the regions shaded in gray are used in the ASAXS experiments.

different phases in the sample and hence the possibility to determine phase compositions. ${ }^{15-18}$ The contrast variation in ASAXS is due to energy dependency of the atomic scattering factor $f(E)$, in particular, in the vicinity of x-ray absorption edges. The atomic scattering factor $f(E)$ can be written as

$$
f(E)=f_{0}+f^{\prime}(E)+i f^{\prime \prime}(E)
$$

with the atomic form factor $f_{0}=Z$ (atomic number), $f^{\prime}(E)$ and $f^{\prime \prime}(E)$ being additional anomalous contributions. The factors $f^{\prime}(E)$ and $f^{\prime \prime}(E)$ are connected to each other via Kramers-Kronig relations. Both factors can be calculated for free atoms using the method of Cromer and Liberman. ${ }^{19}$ The effective electron densities $\eta_{p}$ and $\eta_{m}$ are linear correlated with the atomic scattering factor $f(E)$. A closer view will be given in Sec. IV.

In the present work, we determined chemical compositions of the nanocrystals and the remaining glass matrix from combined ASAXS measurements at the following experimentally accessible x-ray absorption edges: Er L3 (8358 eV), Yb L3 (8944 eV), Pb L3 (13035 eV), and Cd K $(26711 \mathrm{eV})$. In Fig. 1 the energy dependency of the atomic scattering factors (pure elements) is shown. In the vicinity of X-ray absorption edges the $f(E)$ decreases up to $20 \%$ of the normal value (the atomic number). Due to this decrease the differential scattering cross section $d \sigma(q) / d \Omega$ is energy dependent.

\section{EXPERIMENTAL TECHNIQUES}

Nanoglass ceramics with the synthetic composition $21.1 \% \mathrm{SiO}_{2} \quad 6.5 \% \mathrm{~B}_{2} \mathrm{O}_{3} \quad 7.0 \% \mathrm{Al}_{2} \mathrm{O}_{3} \quad 21.0 \% \mathrm{PbF}_{2} \quad 14.3 \% \mathrm{CdF}_{2}$

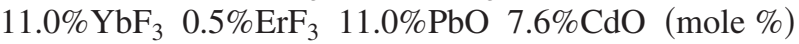
were prepared in a covered platinum crucible. The obtained glass was cut into pieces and heat treated for $0.1-16 \mathrm{~h}$ at 678 $\mathrm{K}$ in order to obtain transparent nanoglass ceramics. Finally, the samples were polished to a thickness of approximately $30 \mu \mathrm{m}$. X-ray absorption measurements were made to determine the accurate thickness. Here, we report the studies on a sample which was annealed at $678 \mathrm{~K}$ for $30 \mathrm{~min}$. The thick- ness determined by the measured x-ray absorption and measured overall composition was found to be $31.5 \pm 0.7 \mu \mathrm{m}$.

The overall composition of the glass was measured using three different methods. The $\mathrm{B}_{2} \mathrm{O}_{3}$ content was determined by inductively coupled plasma mass spectrometry. ${ }^{20}$ The $\mathrm{F}$ content was measured by an ion-selective electrode. ${ }^{21}$ The content of all other elements was determined by x-ray fluorescence spectrometry. ${ }^{22}$

ASAXS experiments were made at four $\mathrm{x}$-ray absorption edges: Er L3 (8358 eV), Yb L3 (8944 eV), Pb L3 $(13035 \mathrm{eV})$, and $\mathrm{Cd} \mathrm{K}(26711 \mathrm{eV})$. At each of these absorption edges five energies below the edge energy were chosen. In addition, four more energies were taken from a region far from any x-ray absorption edges. The combined ASAXS experiments were made at three different beamlines: at the 7T-MPW-SAXS beamline at the synchrotron BESSY II of the Helmholtz-Zentrum Berlin, ${ }^{23}$ at the ID01 beamline at the European Synchrotron Radiation Facility ESRF in France, ${ }^{24}$ and at the B1 beamline at HASYLAB, DESY at Hamburg in Germany. ${ }^{25}$ The $\mathrm{X}$-ray energies were chosen so that the variation in the anomalous correction factors $f^{\prime}(E)$ of the atomic scattering factors $f(E)$ are almost equidistant. While doing so the change in the scattering contrast is expected to be partially equidistant. This is helpful during the analysis of the energy dependence of the scattering contrast.

The two-dimensional (2D) scattering patterns were corrected for transmission, incoming photon flux, detector dead time, detector sensitivity, and solid angle. Furthermore, a geometrical correction was applied so that the measured intensities were projected onto the surface of a sphere with a radius equal to the sample-to-detector distance. After these corrections the scattering contributions of the corresponding empty scattering (no sample in the beam) were subtracted. The corrected 2D scattering patterns were circularly averaged to obtain one-dimensional (1D) scattering curves. This step was possible because the 2D scattering patterns were isotropic, indicating that the particles were on average randomly oriented.

The scattering vector magnitude $q$ was calibrated using silver behenate. The position of the first peak of silver behenate ${ }^{26}$ is at $q=1.076 \mathrm{~nm}^{-1}$. The calibration of the relative scattering intensities to differential scattering cross sections in per centimeter was carried out by using the secondary standard sample glassy carbon, which has been previously calibrated to absolute scattering units by using the isotropic scattering of water. The advantage of the glassy carbon is that it scatters much stronger than water and its intensity is comparable to the sample intensity.

\section{RESULTS}

Figure 2(a) shows the measured and absolute calibrated differential scattering cross sections as a function of the scattering vector length in per nanometer and as a function of $\mathrm{x}$-ray energy. At the white layered regions no experimental data were taken. An increase $(\mathrm{Cd} \mathrm{K})$ or a decrease (valid for all other absorption edges) can be seen close to the absorption edge energies. Measured differential scattering cross sections at five energies below the Yb L3 absorption edge 

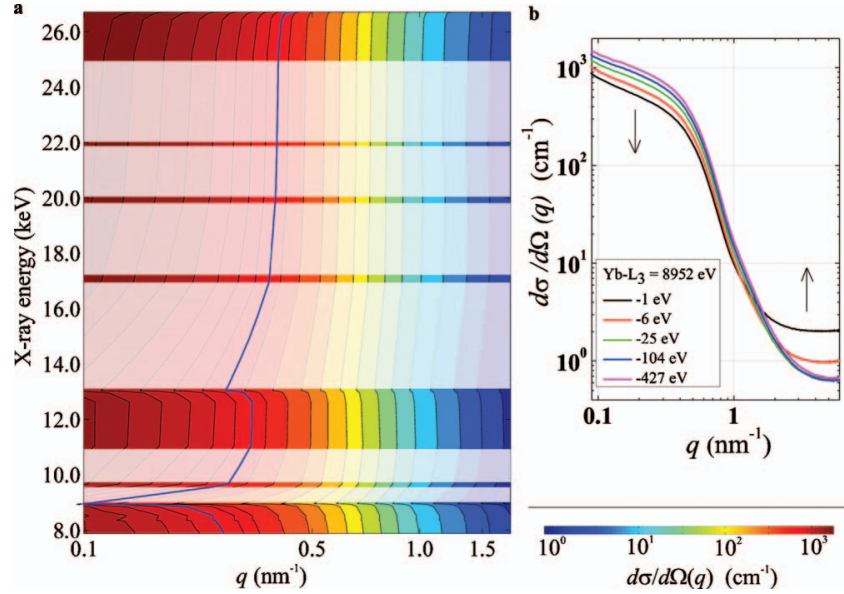

FIG. 2. (Color) Energy dependence of the differential scattering cross sections. (a) Differential scattering cross section of the sample annealed at $678 \mathrm{~K}$ for $30 \mathrm{~min}$. For the white layered regions no data have been taken and the isolines of constant differential scattering cross section are linearly interpolated in these regions. The blue line highlights an isoline of constant differential scattering cross section. A change in the isoline in the vicinity of an absorption edge to smaller $q$ values while increasing energy means a decrease in the scattering contrast and vice versa. (b) Measured scattering curves at five different energies close to the Yb L3 edge. The arrows indicate the change of the scattering contrast when the energy increases close to the particular absorption edge.

are shown for clarity in Fig. 2(b). At low $q$ the intensity decreases as the energy increases to approach the Yb L3 absorption edge. This effect can be understood by the energy dependence of the effective electron density of $\mathrm{Yb}$ in that region, see Fig. 1. At high $q$ the opposite behavior is observed. This effect is well known and is caused by upcoming resonant-Raman scattering close to the absorption edge energy. These two effects are of minor interest in analyzing the anomalous scattering. They will be treated as an additional energy-dependent background contribution to the total scattering intensity.

The first step in data analysis is the determination of the shape and size distribution of the nanoparticles. We have assumed spheroid-shaped particles with a log-normal size distribution based on TEM images of similar glass ceramics (see Fig. 3).

In Fig. 3 two TEM images are shown. Both TEM images show crystals which are locally arranged in a dendritelike structure. The TEM images were taken from two glass samples, one with a higher and the other with a lower amount of $\mathrm{YbF}_{3}$ than the investigated glass ceramic. The glass with a lower amount of $\mathrm{YbF}_{3}$ contains smaller crystals and a smaller volume fraction of particles [Fig. 3(a)] while the system with a higher amount of $\mathrm{YbF}_{3}$ [Fig. 3(b)] shows larger crystals and a higher volume fraction of those crystals. A TEM from the glass with exactly the same composition as the presented glass was not taken so far but it is expected that the structure is similar.

The dendritelike structure of particles can be approximated by spheroid-shaped particles. The differential scattering cross section of spheroid-shaped particles in a matrix can be described by the following equation:
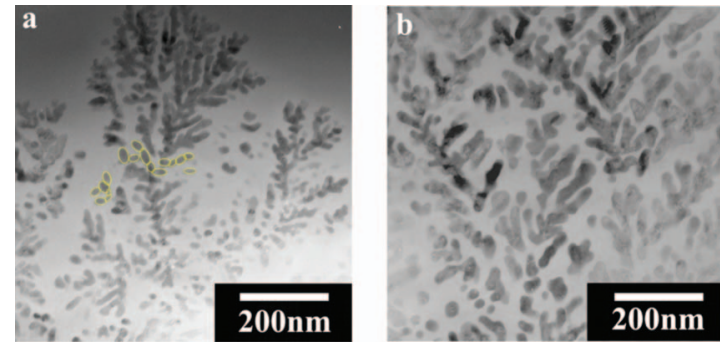

FIG. 3. (Color) TEM visualizations are shown of two similar glass ceramics as the investigated glass ceramic by TEM. The glass (a) contains less $\mathrm{YbF}_{3}$ while glass (b) contains more $\mathrm{YbF}_{3}$ than the investigated glass ceramic. In both cases the crystals are locally arranged in a dendritelike structure. In image (a) the approximation by spheroid-shaped particles used for the ASAXS modeling is made clear.

$$
\begin{aligned}
\frac{d \sigma}{d \Omega}(q)= & |\Delta \rho|^{2} \int_{0}^{\infty} d r \int_{0}^{\pi / 2} d \theta\left\{P(r) F(q, r, \zeta, \theta)^{2}\right. \\
& \left.\times V(r, \zeta)^{2} S(q, r, \zeta, \theta) \cos (\theta)\right\}+B(q) .
\end{aligned}
$$

The $F(q, r, \zeta, \theta)$ is the form factor of spheroid-shaped particles $^{27}$ and is given by

$$
\begin{gathered}
F(q, r, \zeta, \theta)=3 \frac{\sin (q r K)-q r K \cos (q r K)}{(q r K)^{3}}, \\
K(\zeta, \theta)=\sqrt{\cos (\theta)^{2}+\zeta^{2} \sin (\theta)^{2}} .
\end{gathered}
$$

Here, $r$ is the radius of the two equal half axes and $\zeta r$ is the radius of the third half axis. The second integral over $d \theta$ from 0 to $\pi / 2$ in Eq. (3) is the averaging over the orientations. The volume of a spheroid is $V(r, \zeta)=4 / 3 \pi r^{3} \zeta$.

Growth processes in amorphous matrices often produce a log-normal size distribution for the particle dimension. ${ }^{28,29}$ Therefore, a normalized log-normal distribution with the mean size parameter $\mu$ and width parameter $\sigma$ was used

$$
P(r, \mu, \sigma)=\frac{1}{\sqrt{2 \pi} \sigma r} \exp \left\{-\frac{[\ln (r)-\ln (\mu)]^{2}}{2 \sigma^{2}}\right\} .
$$

The scattering background $B(q, E)$ can by written as

$$
B(q, E)=c_{0}(E)+c_{1}(E) q^{-c_{2}(E)},
$$

where the parameters $c_{0}(E), c_{1}(E)$, and $c_{2}(E)$ can be energy dependent. The first term in Eq. (7) describes the scattering caused by thermodynamic fluctuations of the electron density, fluorescence, and resonant-Raman scattering. The second term, that is proportional to $q^{-c_{2}(E)}$, describes the contribution to the scattering intensities originating from surface roughness and large scale correlations of the dendrite structures. Furthermore, due to the relatively small transmission (less than $5 \%$ for all measured energies) it also covers the effect of multiple scattering, the strength of which depends on the transmitted amount of $\mathrm{x}$ rays and, therefore, on the $\mathrm{x}$-ray energy. The $c_{2}(E)$ varies from 2.0 for the low x-ray energies up to 4.0 for the higher $\mathrm{x}$-ray energies.

First attempts to fit the experimental curves with Eq. (3) by neglecting the structure factor $S(q, r, \zeta, \theta)$, low-density 
approximation, resulted in structured reduced residuals in the entire $q$ range. The residuals showed a harmonic modulation, which indicates that the low-density approximation is inappropriate. Therefore, a structure factor was needed to take into account the correlation of the spheroid-shaped particles. Moreover, the TEM images also imply a local high volume fraction of the particles. The structure factor for hard spheres ${ }^{30,31}$ was used by redefining the repulsion radius $r_{\mathrm{p}}$ as a function of the radius $r$ of the smaller half axes of the spheroid particles,

$$
r_{\mathrm{p}}=r[\alpha+\beta K(\zeta, \theta)]
$$

Here, $\alpha$ and $\beta$ are adjustment parameters to reduce or enlarge the repulsion area around the particle. The $K(\zeta, \theta)$ is given by Eq. (5). Due to the expected local high volume fraction $(>20 \%)$ the local monodisperse approximation ${ }^{32,33}$ was used to implement the structure factor in Eq. (3). From the statistical point of view we obtained the best-fit results by using a repulsion radius which equals the smaller half axes $(\alpha \approx 1$ and $\beta=0)$. Such small repulsion radius allows the spheroids to quasi overlap on the tails of the long sides. Therefore, they can build up dendrite structures as seen in the TEM images. The assumed theoretical model has nine adjustable parameters per scattering curve. It follows that we would need 216 parameters to describe all 24 scattering curves recorded at different $\mathrm{x}$-ray energies, when one analyzes the curves separate. This large number of free model parameters can be reduced at least by a factor of 2 by performing a simultaneous regression of all curves at once. In this case the parameters describing the size (mean $\mu$, width $\sigma$, and ratio of the axes $\zeta$ ) of the nanoparticle as well as the repulsion radius $r_{\mathrm{p}}$ and the volume fraction are energy independent and therefore they must be the same for each scattering curve. Finally, the number of free parameters that have to be determined by the simultaneous regression is reduced to 101 parameters. This is effectively equivalent to 4.2 parameters per curve that is an acceptable value from a statistical point of view. The simultaneous nonlinear regression was done by redefining the $1 \mathrm{D}$ minimization problem in $q$ space into a $2 \mathrm{D}$ minimization problem in $q$ and energy space. The $\chi^{2}$ that was minimized is given by

$$
\chi^{2}=\sum_{q} \sum_{E}\left[\frac{\frac{d \sigma}{d \Omega}(q, E)-\frac{d \sigma}{d \Omega}(q, E)_{\exp }}{\delta(q, E)_{\exp }}\right]^{2},
$$

where the sum has to be taken over all $q$ values and all measured energies $E$. The $\delta(q, E)_{\exp }$ is the absolute error of the measured differential scattering cross section at scattering vector $q$ and x-ray energy $E$. As an example two of 24 simultaneously fitted scattering curves and their normalized residuals are shown in Fig. 4(a). The structural model describes the measured intensities within the error band. The normalized residuals are randomly distributed over the whole scattering vector range and they are within $[-1,1]$ and unstructured. The jump at $q=0.7 \mathrm{~nm}^{-1}$ is an artifact of the merging process of two scattering curves measured at two different sample-to-detector distances. Due to the expected strong correlation of the polydispersity $\sigma$ and the eccentricity a

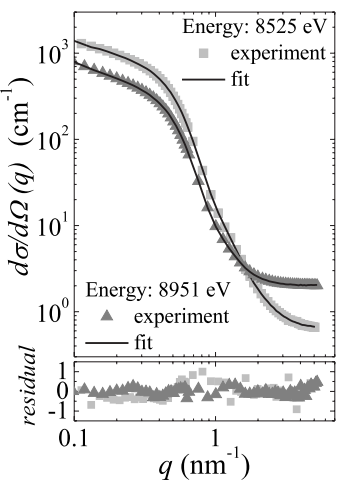

b

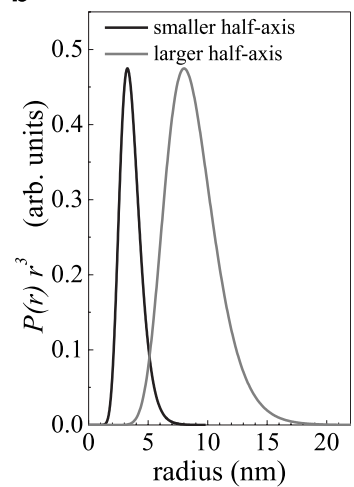

FIG. 4. Visualization of the nonlinear regression and obtained size distribution. (a) The comparison of experimental data (square $=8525 \mathrm{eV}$ and triangle $=8951 \mathrm{eV}$ ) and the fitted theoretical model (solid lines) is shown. The reduced residuals are shown in the bottom part. (b) The volume weighted size distribution for the half axes of the spheroid-shaped particles (solid line $=$ smaller axes and dash line larger axis) is shown.

$\zeta$, we performed a sequence of simultaneous regressions using different starting parameters for both $\sigma$ and $\zeta$. We tested a mesh of those parameters: $\sigma_{\text {start }}=0.1-0.45(\Delta=0.05)$ and $\zeta_{\text {start }}=0.1-3.5(\Delta=0.15)$. If there is a nearly absolute correlation between both parameters all solutions for the parameters $\sigma$ and $\zeta$ have to be randomly distributed. We found that there are two solutions, which have nearly the same reduced $\chi^{2}$. Both solutions have a $\sigma$ of $0.22 \pm 0.01$. On the other hand, two different solutions for the $\zeta$ parameter were found, describing the inverse problem. One solution is $\zeta$ $=2.77 \pm 0.05$ while the other is $\zeta=0.36 \pm 0.01$, the prolate and oblate spheroid, respectively $(1 / 2.77 \approx 0.36)$. We cannot distinguish between oblate or prolate but we can determine the length of the axes and the polydispersity at the same time. The length of the smaller axis was found to be $6.4 \pm 1.4 \mathrm{~nm}$ while the larger axis was found to be $17.7 \pm 3.9 \mathrm{~nm}$. The relatively large errors of the lengths includes the polydispersity of $22 \%$. These dimensions found by simultaneous nonlinear regression are in agreement with a previous XRD study, ${ }^{34}$ which gave coherence lengths of about $20 \pm 3 \mathrm{~nm}$. The corresponding log-normal size distributions of the half axes of the spheroids are shown in Fig. 4(b). From the TEM images we cannot say if the particles are more oblate or prolate. However, the spheroid model is an approximation of the real particle shape, which successfully reproduced the scattering curves. Importantly, we found that the fitted scattering contrast $\Delta \rho(E)^{2}$ is the same for both models.

\section{DISCUSSION}

Figure 5 shows the obtained scattering contrasts as a function of the energy [circles $=7 \mathrm{~T}-\mathrm{MPW}-\mathrm{SAXS}$ beamline BESSY II, ${ }^{23}$ triangles $=$ ID01 beamline ESRF, ${ }^{24}$ and squares =B1 beamline HASYLAB (Ref. 25)]. The decreases or increase in the contrast are visible in the vicinity of the absorption edges. Interestingly, the scattering contrast increases at 


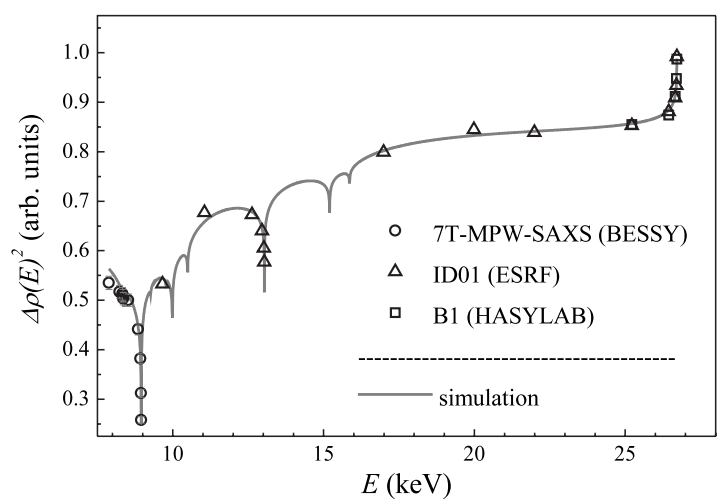

FIG. 5. Energy dependence of the scattering contrast. The circles, squares, and triangles are values obtained from the simultaneous nonlinear regression of all measured scattering curves. The solid line is the simulation using the best-fit parameters with a $\Delta E=1 \mathrm{eV}$.

the $\mathrm{Cd} \mathrm{K}$ edge in contrast to all other absorption edges that were measured. This behavior can be explained within the two-phase approximation by a lower-density phase enriched in $\mathrm{Cd}$ but depleted in $\mathrm{Pb}, \mathrm{Yb}$, and Er. From the scattering contrasts (see Fig. 5) the compositions of the two phases can be determined by a nonlinear regression using the following equation:

$$
\Delta \rho(E)^{2}=N_{\mathrm{fac}} \Delta \eta(E)^{\star} \Delta \eta(E) .
$$

The scattering contrast is the square modulus of the electrondensity difference between the two phases, which is given by

$$
\Delta \eta(E)=\rho_{\mathrm{p}}^{\mathrm{mac}} \sum_{i} \frac{f_{i}\left(E-\delta_{i}\right) x_{\mathrm{p}}^{i}}{M_{i}}-\rho_{\mathrm{m}}^{\mathrm{mac}} \sum_{j} \frac{f_{j}\left(E-\delta_{j}\right) x_{\mathrm{m}}^{j}}{M_{j}} .
$$

The indexes $i$ and $j$ correspond to the different elements in the sample $(\mathrm{O}, \mathrm{Si}, \mathrm{B}, \mathrm{F}, \mathrm{Al}, \mathrm{Cd}, \mathrm{Pb}, \mathrm{Er}$, and $\mathrm{Yb})$. The $\rho_{\mathrm{p}, \mathrm{m}}^{\mathrm{mac}}$ is the macroscopic density of the particle (p) or matrix (m) phase, respectively. The $\delta_{i}$ are chemical shifts of the x-ray absorption edges measured at the sample compared to the theoretical energy of free atoms. The compositions of the particle and matrix phases are given by molar fractions $x_{\mathrm{p}}^{i}$ and $x_{\mathrm{m}}^{i} . M_{i}$ in Eq. (11) is the molar mass of the $i$ th element. The $N_{\text {fac }}$ in Eq. (10) is a normalization factor, which includes the number of particles and a constant value for unit conversion. The regression routine we developed includes penalty functions $^{35}$ to conserve the macroscopic density of the sample $\left(6.338 \mathrm{~g} / \mathrm{cm}^{3}\right)$ and the overall composition of the sample. The compositions inside the particle and glass phase were not constrained otherwise. The overall composition was measured separately by x-ray fluorescence spectroscopy. The regression was done by using theoretical $f(E)$ values given by Cromer and Liberman. ${ }^{19}$ To check the reliability and stability of the nonlinear regression solution we repeated the fit several times using different starting parameters. All tested starting parameters gave same solutions within the calculated errors. Calculation of errors for the compositions using the covariance matrix from the fitting routine underestimates the errors. The variations in the compositions for the different starting parameters give a better estimation of the errors. Therefore, the error is about $10 \%$ for the compositions. The best-fitting parameters are listed in Table I. The solid line in Fig. 5 shows the scattering contrast calculated from the bestfitting parameters. The anomalous effects near the measured absorption edges are well described. Moreover, the calculation shows that at the L2 and L1 absorption edges anomalous effects must be detectable also.

In Fig. 6 a simulation of the scattering intensities using the obtained parameters is shown as a function of the length of the scattering vector $q$ and energies from 7880 to 26711 $\mathrm{eV}$. The anomalous scattering effects in the vicinity of all

TABLE I. Composition of the studied nanoglass ceramic and the best-fitting parameters of the compositions of the phases. The compositions are given in molar fraction. The relative errors of the obtained compositions of the phases are about $10 \%$. The last column shows the chemical shift of the absorption edge energy of the corresponding element relative to pure metal foil. Therefore, one main result is that the

\begin{tabular}{|c|c|c|c|c|c|}
\hline & \multicolumn{2}{|c|}{ Overall composition } & \multicolumn{2}{|c|}{ Obtained compositions by ASAXS } & \multirow[b]{2}{*}{ Chemical shift } \\
\hline & As prepared & As analyzed & Particle & Glass matrix & \\
\hline $\mathrm{B}$ & 0.0407 & 0.0325 & & 0.03 & \\
\hline $\mathrm{O}$ & 0.3163 & 0.4253 & & 0.45 & \\
\hline $\mathrm{F}$ & 0.3288 & 0.2518 & 0.64 & 0.23 & \\
\hline $\mathrm{Al}$ & 0.0438 & 0.0341 & & 0.04 & \\
\hline $\mathrm{Si}$ & 0.0657 & 0.0516 & & 0.06 & \\
\hline $\mathrm{Cd}$ & 0.0685 & 0.0701 & & 0.07 & $\mathrm{Cd} \mathrm{K}: 2.0 \mathrm{eV}$ \\
\hline $\mathrm{Pb}$ & 0.1001 & 0.0977 & 0.17 & 0.09 & $\mathrm{~Pb} \mathrm{~L} 3: 0.0 \mathrm{eV}$ \\
\hline Er & 0.0016 & 0.0015 & 0.02 & & Er L3: $4.0 \mathrm{eV}$ \\
\hline $\mathrm{Yb}$ & 0.0344 & 0.0355 & 0.17 & 0.03 & Yb L3: $7.5 \mathrm{eV}$ \\
\hline Density $\left(\mathrm{g} / \mathrm{cm}^{3}\right)$ & & $6.338 \pm 0.005$ & $7.45 \pm 0.20$ & $6.26 \pm 0.15$ & \\
\hline Volume fraction & & & $6.4 \pm 0.9 \%$ & & \\
\hline
\end{tabular}
nanocrystals have the composition $\mathrm{Pb}_{17} \mathrm{Yb}_{17} \mathrm{Er}_{2} \mathrm{~F}_{64}$. 


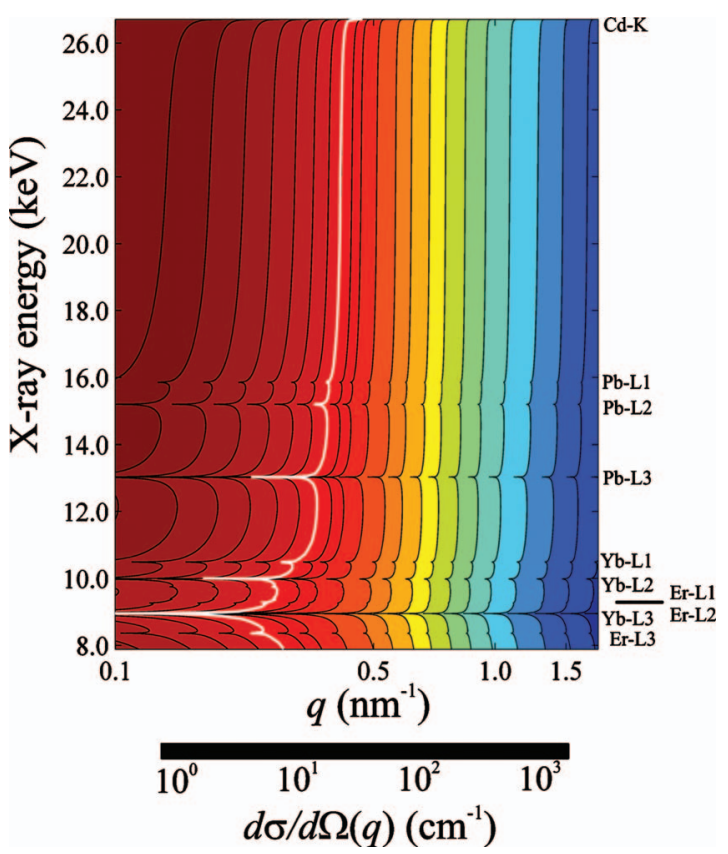

FIG. 6. (Color) A simulation of the energy dependence of the differential scattering cross section is shown. For the calculations the best-fitting parameters were used. The white line marks an isoline of constant differential scattering cross section. The anomalous effects at all x-ray absorption edges (labeled on the right side) are clearly visible.

$\mathrm{X}$-ray absorption edges in that energy range are visible. The simulation agrees well with the measured behavior (see Fig. 2 ) in the regions where data were taken.

\section{CONCLUSION}

From the compositions, which were derived from ASAXS without posing any constrain except the overall composition and density, it is obvious that the nanocrystals have the chemical formula $\mathrm{Pb}_{17} \mathrm{Yb}_{17} \mathrm{Er}_{2} \mathrm{~F}_{64}$, which remarkably corresponds to the crystal phase previously suggested by Kukkonen et al. ${ }^{13}$ Furthermore, no glass components such as $\mathrm{Si}$, $\mathrm{Al}, \mathrm{O}$, or $\mathrm{B}$ are incorporated within the particles, contrary to the EDX analysis by Kukkonen et al. This difference is most likely due to the nature of the characterization techniques and sample preparation. HRTEM with energy dispersive spectroscopy probes a comparatively small volume of the sample (local information). ASAXS delivers an average over a large volume. Additionally, the influence of the sample preparation technique is smaller for ASAXS compared to HRTEM. Finally, we conclude as a main result that the crystalline phase contains neither $\mathrm{Cd}$ nor any light elements. The frequency upconversion process is related with transitions between energy levels of $\mathrm{Yb}$ and Er. We conclude from the arrangement of cadmium that it plays no or at most a minor role in the optical properties of such oxyfluoride glass ceramics. However, it is not certain whether cadmium is needed for the crystallization process of the optically active nanoparticles.

\section{ACKNOWLEDGMENTS}

We acknowledge the workers at the Central laboratory of Saint-Gobain Spain (Aviles) for the overall composition analysis of the glass ceramic. We thank Thomas Höche, Institut für Oberflächenmodifikation, Leipzig, Germany for the TEM images. We thank Klaus Effland, Helmholtz-Zentrum Berlin für Materialien und Energie, for technical support. *sylvio.haas@ helmholtz-berlin.de

${ }^{1}$ F. Auzel, Chem. Rev. 104, 139 (2004).

${ }^{2}$ X. Sun, M. Gu, S. Huang, X. Jin, X. Liu, B. Liu, and C. Ni, J. Lumin. 129, 773 (2009).

${ }^{3}$ F. Weng, D. Chen, Y. Wang, Y. Yu, P. Huang, and H. Lin, Ceram. Int. 35, 2619 (2009).

${ }^{4}$ M. Takahashi, M. Izuki, R. Kanno, and Y. Kawamoto, J. Appl. Phys. 83, 3920 (1998).

${ }^{5}$ H. Yu, K. Zhou, K. Chen, J. Song, C. Hou, and L. Zhao, J. Non-Cryst. Solids 354, 3649 (2008).

${ }^{6}$ Z. Yu, Q. Yang, C. Xu, and Y. Liu, Mater. Res. Bull. 44, 1576 (2009).

${ }^{7}$ S. León-Luis, J. Abreu-Afonso, J. Peña-Martínez, J. MéndezRamos, A. C. Yanes, J. del-Castillo, and V. D. Rodríguez, J. Alloys Compd. 479, 557 (2009).

${ }^{8}$ E. Gagnon, P. Ranitovic, X. Tong, C. Cocke, M. Murname, H. Kapteyn, and A. Sandhu, Science 317, 1374 (2007).

${ }^{9}$ M. Digonnet, Rare-Earth-Doped Fiber Lasers and Amplifiers (Marcel Dekker, New York, 2001).

${ }^{10}$ P. Tick, N. Borrelli, L. Cornelius, and M. Newhouse, J. Appl. Phys. 78, 6367 (1995).

${ }^{11}$ W. Pisarski, J. Pisarski, R. Liesoecki, L. Grobelny, G. DominiakDzik, and W. Ryba-Romanowski, Chem. Phys. Lett. 472, 217
(2009).

${ }^{12}$ Y. Wang and J. Ohwaki, Appl. Phys. Lett. 63, 3268 (1993).

${ }^{13}$ L. Kukkonen, I. Reaney, D. Furniss, M. Pellatt, and A. Seddon, J. Non-Cryst. Solids 290, 25 (2001).

${ }^{14}$ A. Guinier and G. Fournet, Small-Angle Scattering of X-rays (Wiley, New York, 1955).

${ }^{15}$ G. Goerigk, H. Haubold, O. Lyon, and J. Simon, J. Appl. Crystallogr. 36, 425 (2003).

${ }^{16}$ A. Hoell, F. Bley, A. Wiedenmannm, J. Simon, P. Mazuelas, and P. Boesecke, Scr. Mater. 44, 2335 (2001).

${ }^{17}$ S. Haas, A. Hoell, G. Zehl, I. Dorbandt, P. Bogdanoff, and S. Fiechter, ECS Trans. 6, 127 (2008).

${ }^{18}$ G. Zehl, G. Schmithals, A. Hoell, S. Haas, C. Hartnig, I. Dorbandt, P. Bogdanoff, and S. Fiechter, Angew. Chem., Int. Ed. 46, 7311 (2007).

${ }^{19}$ D. Cromer and D. Liebermann, J. Chem. Phys. 53, 1891 (1970).

${ }^{20} \mathrm{H}$. Taylor, Inductively Coupled Plasma Mass Spectrometry (Academic Press, New York, 2001).

${ }^{21}$ A. Bard and L. Faulkner, Electrochemical Methods: Fundamentals and Applications (Wiley, New York, 2000).

${ }^{22}$ B. Beckhoff, B. Kanngiesser, N. Langhoff, R. Wedell, and H. Wolff, Handbook of Practical X-Ray Fluorescence Analysis (Springer, New York, 2006). 
${ }^{23}$ A. Hoell, I. Zizak, H. Bieder, and L. Mokrani, German Patent No. DE 102006029449 (2006).

${ }^{24}$ S. Lequien, L. Goirand, and F. Lesimple, Rev. Sci. Instrum. 66, 1725 (1995).

${ }^{25}$ H. Haubold et al., Rev. Sci. Instrum. 60, 1943 (1989).

${ }^{26}$ K. Binnemans, R. V. Deun, B. Thijs, I. Vanwelkenhuysen, and I. Geuens, Chem. Mater. 16, 2021 (2004).

${ }^{27}$ A. Guinier, Ann. Phys. (Paris) 12, 161 (1939).

${ }^{28}$ L. Kiss, J. Soederlund, G. Niklasson, and C. Granqvist, Nano- struct. Mater. 12, 327 (1999).

${ }^{29}$ R. Bergmann and A. Bill, J. Cryst. Growth 310, 3135 (2008).

${ }^{30}$ J. Pedersen, J. Appl. Crystallogr. 27, 595 (1994).

${ }^{31}$ J. Percus and G. Yevick, Phys. Rev. 110, 1 (1958).

${ }^{32}$ A. Vrij, J. Chem. Phys. 71, 3267 (1979).

${ }^{33}$ J. Pedersen, Adv. Colloid Interface Sci. 70, 171 (1997).

${ }^{34}$ R. Wurth, M.S. thesis, Friedrich-Schiller-University Jena, 2007.

${ }^{35}$ J. Zhang and X. Zhang, J. Optim. Theory Appl. 118, 635 (2003). 